\title{
The Theory and Implementation of Bayesian Networks Structural Learning using Tabu Search Algorithm
}

\author{
Najib Rahman, Ismaeil Rabbani, Reyhaneh Rafiei, Maryam Asadi * \\ Department of Computer Science, University of Kabul, Afghanistan
}

\section{Keywords}

Graphical Models,

Bayesian Networks,

Structural learning,

Machine learning,

Tabu Search

\begin{abstract}
This paper portrays the hypothesis and execution of Bayesian systems basic getting the hang of utilizing unthinkable pursuit calculation. Bayesian systems give an extremely broad but powerful graphical language for calculating joint likelihood disseminations. Finding the ideal structure of Bayesian systems from information has been demonstrated to be NP-hard. In this paper, unthinkable hunt has been created to give progressively proficient structure. We actualized auxiliary learning in Bayesian systems with regards to information characterization. With the end goal of correlation, we considered order task and applied general Bayesian systems alongside this classifier to certain databases. Our trial results show that the Tabu pursuit can locate the great structure with the less time multifaceted nature. The reenactment results affirmed that utilizing Tabu hunt so as to discover Bayesian systems structure improves the grouping exactness.
\end{abstract}

\section{Introduction}

A Bayesian system (BN) comprises of a coordinated non-cyclic diagram $G$ and a set $P$ of likelihood dispersions, where hubs and circular segments in $G$ speak to irregular factors and direct connections between's factors individually, and $\mathrm{P}$ is the arrangement of nearby circulations for every hub. Learning BNs has become a functioning examination in the previous decade (1-5). The objective of learning a BN is to decide both the structure of the system (structure learning) and the arrangement of contingent likelihood tables CPTs (parameter learning). Learning the structure, or causal conditions, of a graphical model of likelihood, for example, a Bayesian system is frequently an initial phase in Tabu under vulnerability. The structure of a Bayesian system encodes variable independencies. Since the quantity of potential structures is incredibly tremendous, structure adapting regularly has high computational multifaceted nature. Finding the ideal structure of a BN from information has been demonstrated to be NP-hard. Subsequently, heuristic and inexact learning calculations are the sensible arrangement. Applicability and efficiency of BN in different fields of engineering are investigated as some can be found in these works $(1,2,3,4)$. Thusly, avaricious scorebased calculations have been created to give increasingly proficient structure learning at an exactness tradeoff. Unthinkable Search, is a local inquiry, implies we locate the following conceivable state from the arrangement of neighborhood states and we will at that point choose to move to that state or not (6-11). With a Tabu hunt it isn't unexpected to recollect the best move seen during the total pursuit and return that as the best arrangement. The primary guideline behind Tabu inquiry is that it has some memory of the states that it has just been examined and it doesn't return to those states. Because of the qualities of Tabu pursuit, this inquiry calculation was utilized in this paper for learning the structure of Bayesian systems (12-16). The remainder of the paper is composed as follows. In Section 2, we audit the fundamental ideas identified with Bayesian systems. In Section 3, we talk about basic learning in Bayesian systems. Next, Section 4 is depicted the Tabu inquiry calculation. In area 5 we will represent the usage results. At last, Section 6 sums up the fundamental ends. 2. Bayesian Networks The Bayesian strategy is based upon the notable Bayes' Rule, which is itself gotten from the central principle for likelihood analytics (17-22).

Bayes' standard not just makes the way for frameworks that advance probabilities as new proof is procured, yet in addition gives the supporting to the inferential and learning systems utilized in Bayesian systems [4]. A Bayesian system is a coordinated non-cyclic chart (DAG) that gives a smaller portrayal or factorization of the joint likelihood appropriation for a gathering of factors (23-28). Graphically, a BN contains hubs and coordinated edges between those hubs. Since the nonappearance of an edge between two hubs suggests restrictive autonomy, the likelihood dispersion of a hub can be controlled by Tabu about the conveyances of its folks. Along these lines, the joint likelihood appropriation for the whole system can be indicated. This relationship can be caught numerically utilizing the chain rule in Equation 1.

$$
p(x)=\prod_{i=1}^{n} p\left(x_{i} \mid \text { parents }\left(x_{i}\right)\right)
$$

As a rule terms, this condition expresses that the joint likelihood appropriation for hub $\mathrm{x}$ is equivalent to the result of the likelihood of every segment $\mathbf{x}$ I of $\mathbf{x}$ given the guardians of $\mathbf{x} I$. Every hub has a related contingent likelihood table that gives the its likelihood being in a specific state, given any blend of parent states. At the point when proof is entered for a hub in the system, the essential standard for likelihood analytics and Bayes' standard can be utilized to spread this proof through the system, refreshing influenced likelihood disseminations. Proof can be engendered from guardians to youngsters just as from kids to guardians, making this strategy exceptionally powerful for both forecast and analysis (29-31). 3. Basic Learning in Bayesian Networks We are keen on taking in BN from preparing information $\mathrm{D}$ comprising of models $\mathrm{x}$. The two significant errands in learning a BN are: learning the graphical structure, and afterward learning the parameters ( $C P$ table passages) for that structure. The structure of a $\mathrm{BN}$, characterizes the contingent independencies among the factors. There are various methods of building up the Bayesian system structure [9, 4]. Learning structure implies taking in restrictive independencies from perceptions. The parameters of a BN with a given structure are assessed by Maximum Likelihood (ML) or Maximum a Posteriori (MAP) estimation. One can 
utilize a comparable technique for picking a structure for the BN that fits to the watched information. Be that as it may, basic taking in is somewhat not quite the same as parameter learning. In the event that we think about ML estimation or MAP estimation with uninformative priors, at that point the structure that amplifies the probability will be the outcome. For this situation complex structures (for example that has more conditions between factors) will be increasingly competent to improve the probability since they have more level of opportunity. Notwithstanding, the point is to discover a structure that encodes the independencies among the factors, that is we need our structure to be as straightforward as could be expected under the circumstances (3236). The basic way to deal with this issue is to present a scoring capacity that assesses each system as for the preparation information, and afterward to scan for the best system. All in all, this advancement issue is obstinate, yet for certain limited classes of systems there are productive calculations requiring polynomial time in the quantity of factors in the system. 3.1. Scoring structure, the rules for choosing a structure have two terms. One for augmenting the probability and one for limiting the multifaceted nature. The log-probability score is a straightforward score. While log-probability score is extremely basic, isn't appropriate for learning the structure of the system, since it will in general kindness complete chart structures (in which each factor is associated with each other variable). So as to abstain from overfitting, we can include a punishment for the multifaceted nature of the system dependent on the quantity of parameters. So as to abstain from overfitting, we can include a punishment for the multifaceted nature of the system dependent on the quantity of parameters. MDI (Minimum Description Length) says that, the best model of an assortment of information is the model that limits the length of the encoding. One of the significant properties of MDL techniques is that they give a characteristic defend against overfitting, on the grounds that it executes a tradeoff between the multifaceted nature of the theory (model class) and the unpredictability of the information given the speculation. Subsequently learning a BN dependent on the MDI score is proportionate to limiting the punishment score for the multifaceted nature of the structure limiting the probability of information given the structure, in light of ML parameter gauges [8]. A third chance is to relegate an earlier conveyance over system structures and locate the most probable system by joining its earlier likelihood with the likelihood agreed to the system by the information. This is the "Bayesian" way to deal with arrange scoring and is called BIC score. Contingent upon the earlier conveyance utilized, it can take different structures. Notwithstanding, genuine Bayesians would average over all conceivable system structures instead of singling out a specific system for forecast. Tragically, this by and large requires a lot of calculation. BIC score results from the guess of the probability of the information given the structure around the MAP or MI evaluations of the parameters, the estimation brings about a similar definition of MDL. Akaike's Information Criterion (AIC) is a proportion of the integrity of attack of an expected measurable model. It is grounded in the idea of entropy. The AIC is an operational method of exchanging off the intricacy of an expected model against how well the model fits the information. 3.2. Looking through structure space Searching the structure space for high scored structures is the following issue in basic learning. It has been indicated that finding the structure with most extreme scoring is NP-hard. Along these lines, for subjective structures, heuristic inquiry calculations are utilized. Bayesian system basic learning calculations contrast for the most part in the manner by which they search through the space of system structures. A few calculations are presented beneath. 3.2.1 K2 Search $\mathrm{K} 2$ is an eager calculation and is introduced with a requesting of hubs to such an extent that the guardians of a hub are recorded over the hub (I. e. topological requesting). Beginning with an unfilled structure and following the hub list all together, one adds a parent to the hub that expands the score. The quantity of guardians to be added to a solitary variable might be restricted to a foreordained consistent for quick induction. $\mathrm{K} 2$ discovers structures rapidly whenever given a sensible requesting $\alpha$. In the event that the quantity of guardians per variable is compelled to a steady upper bound, $\mathrm{K} 2$ has most pessimistic scenario polynomial running time in the number $n$ of factors. Two away from of eagerness are failure to backtrack (i.e., fix the option of a circular segment) or think about the joint impacts of including numerous curves (guardians). This is the reason avaricious structure learning calculations are touchy to the nearness of unessential factors in the preparation information, an inescapable issue in AI. Furthermore, $\mathrm{K} 2$ is especially touchy to the variable requesting in light of the fact that bends neglect to be included, bringing about unexplained connections, at whatever point competitor guardians are assessed in any request that blocks a causal reliance. 3.2.2. Slope Climbing The possibility of a slope climbing search calculation is to create a model in a bit by bit style by making the most extreme conceivable improvement in a target quality capacity at each progression. Introduce with a system structure, potentially irregular, assess the adjustment in the score for all circular segment changes on this system and pick the one that has the most extreme change. Proceed with this procedure until no more circular segment changes increment the score. This calculation for the most part sticks into neighborhood maxima. 3.2.3 Iterated slope climbing the issue of stalling out in neighborhood optima is a major disadvantage of the slope climbing calculation. Different other improvement strategies, for example, iterated slope climbing attempt to conquer this issue. Iterated slope climbing apply neighborhood search until nearby most extreme. Arbitrarily bother the structure and rehash the procedure for some sensible number of emphasess. 4. Unthinkable Search Tabu inquiry is an iterative hunt technique. It utilizes a nearby inquiry calculation at every cycle to look for the best arrangement in some subset of the area, which originated from the best arrangement got at the last emphasis. At every cycle, the neighborhood scan calculation searches for the best improving arrangement. On the off chance that all arrangements are not improving the target work esteem, at that point it searches for the least weakening arrangement. Forbidden hunt keeps a rundown, which is called Tabu rundown, of the moves it used to acquire the best arrangements during every emphasis and to limit the nearby pursuit calculation in reusing those moves. A memory is utilized to monitor this Tabu rundown. Typically, the Tabu rundown has a predetermined length. Accordingly, this rundown shifts from cycles to emphasess. FIFO (first-in-first-out) discipline is utilized to deal with the length of the Tabu rundown. By utilizing the Tabu rundown, Tabu pursuit empowers the acknowledgment of non-improving arrangements in order to escape from nearby ideal. Nonetheless, there is a significant special case to the Tabu rundown. In the event that the Tabu moves to produce a superior arrangement than all the best arrangements acquired up until now, we will acknowledge this Tabu move and free it out of the Tabu rundown. This abrogate of the Tabu rundown is designated "goal conditions" [15]. There are essentially three systems utilized in Tabu hunt: the restricting procedure, the liberating technique, and the momentary methodology. The precluding methodology controls what enters the Tabu rundown. The liberating system chooses what leaves the Tabu rundown and when the leave will happen. The momentary procedure deals with the interchange between the prohibiting methodology and liberating technique to produce and select preliminary arrangements. There are fundamentally five parameters required before running the Tabu hunt calculation. They are the nearby hunt system, the local structure, the Tabu conditions, the yearning conditions, and the halting guideline. The Tabu pursuit calculation will be ended observing some halting principles. The principles might be a fixed number of cycles, a fixed number of CPU time, or a fixed number of continuous emphases without an improvement in the best target work esteem, and so forth. There are some hypothetical examinations on combination prospects of Tabu hunt. Memory Tabu Search unites to the worldwide ideal with likelihood one if haphazardly produced vectors $(x)$ follows Gaussian or uniform dispersion. Focalized Tabu Search merges to the worldwide ideal with likelihood one [18]. Tabu pursuit is demonstrated effective in taking care of numerous troublesome enhancement issues in numerous literary works. By utilizing the Tabu show, it permits the acknowledgment of nonimproving arrangements to maintain a strategic distance from the inclination of being caught into a nearby ideal. Consequently, it is conceivable to locate a worldwide ideal by utilizing the Tabu inquiry. Be that as it may, the worldwide ideal isn't ensured by this strategy. There are a few troubles in actualizing this strategy since at times there are an excessive number of parameters to be resolved. In Figure 1, the pseudo code for Tabu hunt is introduced. Unthinkable Search, is a local pursuit, implies we locate the following conceivable state from 
the arrangement of neighborhood states and afterward we choose if to move to that state. Unthinkable inquiry, considers the arrangement of all conceivable neighborhood states and takes the best one. With a Tabu pursuit it is entirely expected to recall the best move seen during the total hunt and return that as the best arrangement. The fundamental rule behind Tabu pursuit is that it has some memory of the states that it has just researched and it doesn't return to those states. Not returning to recently visited states helps in two different ways. It keeps away from the pursuit getting into a circle by persistently looking through a similar zone without really gaining any ground. Thusly, this enables the pursuit to investigate areas that it may not in any case investigate. The moves that are not permitted to be returned to are held in an information structure (generally a rundown) and these moves are designated "Unthinkable". In Figure 1 Recency is the visited moves (which is now and then called a history) which doesn't as a rule develop until the end of time. On the off chance that we did this, we may limit the inquiry to an extreme. Subsequently, the reason for a Recency work is to confine the size of the rundown here and there. It is all very well saying that we will dismiss any move that is Tabu. In the event that a Tabu move is more prominent than the goal level, at that point we acknowledge the move. The least demanding and most regular execution of this capacity is to just keep the rundown at a fixed size and utilize a FIFO (First-In, FirstOut) calculation to keep up the rundown. Yet, different techniques are here and there actualized, contingent upon the issue. Perhaps the rundown size parameter is dynamic in that it changes as the calculation runs. One strategy for doing this is to keep the rundown little when states are not being rehashed all the time, however when rehashed states continue being created the rundown is made bigger with the goal that the inquiry is compelled to investigate new regions.

\section{Results and Discussion}

Test results We run our investigations on the 3 datasets recorded in the iris dataset (D1), is ostensibly the most popular dataset utilized in information mining, contains 150 models every one of three sorts of plant: Iris setosa, Iris versicolor, and Iris virginica. There are four characteristics: sepal length, sepal width, petal length, and petal width (all deliberate in centimeters). Not at all like past dataset, all characteristics have values that are numeric. The work arrangements dataset (D2) sums up the result of Canadian agreement exchanges in 1987 and 1988. It incorporates every single aggregate understanding came to in the business and individual administrations segment for associations with at any rate 500 individuals (educators, medical caretakers, college staff, police, and so on.). Each case concerns one agreement, and the result is whether the agreement is regarded worthy or unsatisfactory. The D3 dataset incorporates discourse information from Deterding database.

Table 1: Experimental datasets

\begin{tabular}{llccc}
\hline & & $\begin{array}{c}\text { Number of } \\
\text { instances }\end{array}$ & $\begin{array}{c}\text { Number of } \\
\text { attributes }\end{array}$ & $\begin{array}{c}\text { Number of } \\
\text { classes }\end{array}$ \\
\hline Iris & D1 & 150 & 4 & 3 \\
Labor & D2 & 57 & 17 & 3 \\
Vowel & D3 & 1500 & 20 & 7 \\
\hline
\end{tabular}

This database is usually for vowel recognition. Speaker's sex and identity are added to this database as new features. Since the amount of data in all datasets is limited, $\mathrm{n}$-fold cross validation method was used in our experiments.

Table 2: Clustering Performance

\begin{tabular}{ccccc}
\hline & K1 & Hill Climbing & $\begin{array}{c}\text { Iterative Hill } \\
\text { Climbing }\end{array}$ & $\begin{array}{c}\text { Tabu } \\
\text { Search }\end{array}$ \\
\hline D1 & 94 & 94 & 93.33 & 92.67 \\
D2 & 92.98 & 85.96 & 87.72 & 89.47 \\
D3 & 80.60 & 77.47 & 81.11 & 82.23 \\
\hline
\end{tabular}

In $n$-fold cross-validation, information is part to $n$ around equivalen parcels and one by one is utilized for testing and the rest of preparing This methodology is rehashed $\mathrm{n}$ times. In this way, toward the end, each example has been utilized precisely once for testing.10-crease cross-approval was utilized in our examinations. In this manner, the train and test methods are executed a sum of multiple times and the all-out blunder rate is the normal of mistakes in totally directed tests.
We watched arrangement execution for every calculation. The precision of every classifier depends on the level of fruitful forecasts on the test sets of each dataset. In Figure 1 and Table 2, the exactnesses of the four learning strategies talked about in this paper are summed up. Calculations were done on a PC with a $3.20 \mathrm{GHz}$ Pentium CPU, and $1 \mathrm{~GB}$ of RAM memory. In our investigations we utilized BIC scored for scoring the structure.

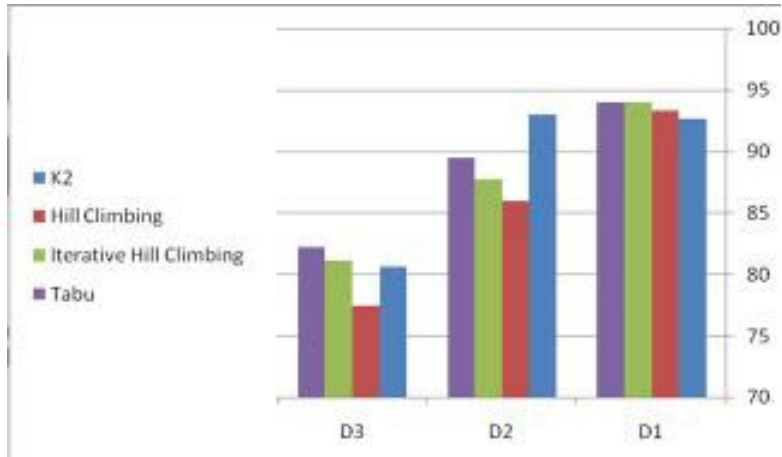

Figure 1: Clustering Performance on datasets D1, D2, and D3

\section{Conclusion}

In this paper, basic learning dependent on Tabu quest was proposed for learning the structure of BN. Some learning calculations including K2, Hill Climbing, and Iterative Hill climbing, were actualized and contrasted with Tabu inquiry. Proposed calculations in this paper show great exhibitions in finding fitting structure, and present a generally low time multifaceted nature. Thus, it was indicated that from the purpose of bunching execution, the Tabu hunt is the best calculation and $\mathrm{K} 2$ is less tedious contrasted with different calculations. In light of the outcomes acquired for order of 3 distinctive datasets, we accept that the Tabu inquiry can be utilized all the more regularly for Bayesian systems auxiliary learning in numerous applications. As future works, we are intrigued to explore the utilization of transformative based calculations for looking through the state space in basic learning.

\section{Declaration of Conflict of Interests}

The authors declare that there is no conflict of interest.

\section{References}

[1.] Adeli, E., Rosic, B.V., Matthies, H.G., Reinstädler, S., and Dinkler, D., Bayesian Parameter Determination of a CT-Test described by a Viscoplastic-Damage Model considering the Model Error. DOI: 10.13140/RG.2.2.26924.82562, 2019.

[2.] Adeli, E., Rosić, B.V., Matthies, H.G., Reinstädler, S., and Dinkler D., Comparison of Bayesian Methods on Parameter Identification for a Viscoplastic Model with Damage. DOI: 10.13140/RG.2.2.30280.26889, 2019

[3.] Adeli, E., Matthies, H.G., Parameter Identification in Viscoplasticity using Transitional Markov Chain Monte Carlo Method. http://arxiv.org/abs/1906.10647, 2019.

[4.] Adeli, E., Rosić, B.V., Matthies, H.G., and Reinstädler S., Effect of Load Path on Parameter Identification for Plasticity Models using Bayesian Methods. http://arxiv.org/abs/1906.07246, 2018.

[5.] Mostafavi SM, Eissazadeh S, Piryaei M. Comparison of Polymer and Ceramic Membrane in the Separation of Proteins in Aqueous Solution Through Liquid Chromatography. Journal of Computational and Theoretical Nanoscience. 2019;16(1):157-64.

[6.] Ahmadipour A, Shaibani P, Mostafavi SA. Assessment of empirical methods for estimating potential evapotranspiration in Zabol Synoptic Station by REF-ET model. Medbiotech Journal. 2019;03(01):1-4.

[7.] Jafari S, Mostafavi SA. Investigation of nitrogen contamination of important subterranean water in the plain. Medbiotech Journal. 2019;03(01):10-2. 
[8.] Z. Man AGE, S. M. Mostafavi, A. Surendar. Fuel oil characteristics and applications: economic and technological aspects. Petroleum Science and Technology. 2019.

[9.] Seyed Mojtaba Mostafavi1 HM, Taskhiri MS. In Silico Prediction of Gas Chromatographic Retention Time of Some Organic Compounds on the Modified Carbon Nanotube Capillary Column. Journal of Computational and Theoretical Nanoscience. 2019;16(01):151-6.

[10.] Mostafavi SM, Ebrahimi A. Mercury determination in work place air and human biological samples based on dispersive liquid-liquid micro-extraction coupled with cold vapor atomic absorption spectrometry. Analytical Methods in Environmental Chemistry Journal. 2019;2(04):49-58.

[11.] Aida Badamchi Shabestari BAA, Maryam Shekarchi, Seyed Mojtaba Mostafavi. Development of Environmental Analysis for Determination of Total Mercury in Fish Oil Pearls by Microwave Closed Vessels Digestion Coupled with ICP-OES. Ekoloji. 2018;27(106):1935.

[12.] Bayat M, Mostafavi SM. Investigation of Interleukin 2 as Signaling Molecule in Human Serum Albumin. The Pharmaceutical and Chemical Journal. 2018;5(02):183-9.

[13.] Pasban A, Mostafavi SM, Malekzadeh H, Mohammad Nazari B. Quantitative Determination of LPG Hydrocarbons by Modified Packed Column Adsorbent of Gas Chromatography Via Full Factorial Design. Journal of Nanoanalysis. 2017;4(1):31-40.

[14.] Somayyeh Heidari MI, Seyed Mojtaba Mostafavi, . A Validated and Rapid HPLC Method for Quantification of Human Serum Albumin in Interferon beta-1a Biopharmaceutical Formulation. MedBioTech Journal. 2017:1(01):29.

[15.] Mostafavi SM, Bagherzadeh K, Amanlou M. A new attempt to introduce efficient inhibitors for Caspas-9 according to structure-based Pharmacophore Screening strategy and Molecular Dynamics Simulations. Medbiotech Journal. 2017;01(01):1-8.

[16.] Amanlou M, Mostafavi SM. In sillico screening to aim computational efficient inhibitors of caspase- 9 by ligandbased pharmacophore modeling. Medbiotech Journal. 2017;01(01):34-41.

[17.] Mostafavi SM, Pasban AA, Piryaei M, Sadeghpour S, Masoumi M, Rouhollahi A, et al. Acidity removal of Iranian heavy crude oils by nanofluid demulsifier: An experimental investigation. Journal of Nanoanalysis. 2017:10-7.

[18.] Parvanian S, Mostafavi SM, Aghashiri M. Multifunctional Nanoparticle Developments in Cancer Diagnosis and Treatmen. Sensing and Bio-Sensing Research. 2016;1(2):22.

[19.] Mostafavi SM, editor Enhancement of mechanical performance of polymer nanocomposites using $\mathrm{ZnO}$ nanoparticles. 5th International Conference on Composites: Characterization, Fabrication and Application (CCFA-5); 2016: Iran University of Science and Technology.

[20.] Abolfazl Davoudiroknabadi SMM, Seyed Sajad Sajadikhah. An Introduction to Nanotechnology. Mikima Book; 2016.

[21.] Abolfazl Davodiroknabadi SMM, Ali Asghar Pasban. Fundamentals of Nanostructure and Nanomaterial. Mikima Book; 2016.

[22.] Mostafavi SM. 3D Graphene Biocatalysts for Development of Enzymatic Biofuel Cells: A Short Review. Journal of Nanoanalysis. 2015;2(2):57-62.

[23.] Seyed Mojtaba Mostafavi MP, Ahmad Rouhollahi, Mohajeri A. Separation and Quantification of Hydrocarbons of LPG Using Novel MWCNT-Silica Gel Nanocomposite as Packed Column Adsorbent of Gas Chromatography. Journal of NanoAnalysis. 2014;1(01):01.

[24.] Seyed Mojtaba Mostafavi MP, Ahmad Rouhollahi, Mohajeri A. Separation of Aromatic and Alcoholic Mixtures using Novel MWCNT-Silica Gel Nanocomposite as an Adsorbent in Gas Chromatography. Journal of NanoAnalysis. 2014;1(01):11.

[25.] Zabihi O, Khodabandeh A, Mostafavi SM. Preparation, optimization and thermal characterization of a novel conductive thermoset nanocomposite containing polythiophene nanoparticles using dynamic thermal analysis. Polymer degradation and stability. 2012;97(1):3-13.

[26.] Shamsipur M, Beigi AAM, Teymouri M, Poursaberi T, Mostafavi SM, Soleimani P, et al. Biotransformation of methyl tert-butyl ether by human cytochrome P450 2A6. Biodegradation. 2012;23(2):311-8.

[27.] Seyed Mojtaba Mostafavi AR, Ali Akbar Miranbeigi. Handbook of Mineral Analysis. Mani Publication, Ltd, Isfahan, Iran; 2012.
[28.] Zabihi O, Mostafavi SM, Ravari F, Khodabandeh A, Hooshafza $\mathrm{A}$, Zare $\mathrm{K}$, et al. The effect of zinc oxide nanoparticles on thermo-physical properties of diglycidyl ether of bisphenol A/2, 2'-Diamino-1, 1'-binaphthalene nanocomposites. Thermochimica acta. 2011;521(1-2):49-58

[29.] Ghasemian MB, Rawal A, Shahrbabaki Z, Zhang Q, Lu T, Liu Y, et al. Evidence of phase coexistence in hydrothermally synthesized $\mathrm{K} 0.5 \mathrm{NaO} 0.5 \mathrm{NbO} 3$ nanofibers. Journal of Materials Chemistry A. 2020.

[30.] Ghasemian MB, Daeneke T, Shahrbabaki Z, Yang J, KalantarZadeh K. Peculiar piezoelectricity of atomically thin planar structures. Nanoscale. 2020;12(5):2875-901.

[31.] Heidari M, Ghasemi S, Heidari R. The Effects of Leadership and Employment in Technical Capabilities of Sport Teams. Journal of Humanities Insights. 2019;3(02):75-80.

[32.] Ghasemian MB, Mayyas M, Idrus-Saidi SA, Jamal MA, Yang J, Mofarah SS, et al. Self-Limiting Galvanic Growth of $\mathrm{MnO} 2$ Monolayers on a Liquid Metal-Applied to Photocatalysis. Advanced Functional Materials. 2019;29(36):1901649.

[33.] Ghasemian MB, Rawal A, Liu Y, Wang D. Approaching Piezoelectric Response of Pb-Piezoelectrics in Hydrothermally Synthesized BiO.5(Na1-xKx)0.5TiO3 Nanotubes. ACS Applied Materials \& Interfaces. 2018;10(24):20816-25.

[34.] Ghasemian MB, Rawal A, Wang F, Chu D, Wang D. Lattice evolution and enhanced piezoelectric properties of hydrothermally synthesised 0.94(Bi0.5Na0.5)TiO3$0.06 \mathrm{BaTiO} 3$ nanofibers. Journal of Materials Chemistry C. 2017;5(42):10976-84.

[35.] Ghasemian MB, Lin Q, Adabifiroozjaei E, Wang F, Chu D, Wang D. Morphology control and large piezoresponse of hydrothermally synthesized lead-free piezoelectric (BiO.5Na0.5)TiO3 nanofibres. RSC Advances. 2017;7(25):150206.

[36.] Anbia M, Ghasemian MB, Shariati S, Zolfaghari G. Employing a new modified nanoporous carbon for extraction and determination of 1,10-phenanthroline and 2,2'-bipyridine by SPE and use of the Taguchi optimization method. Analytical Methods. 2012;4(12):4220-9.

[37.] Samira Eissazadeh SMM, Masoumeh Piryaei,, Taskhiri MS. Application Of Polyaniline Nanostructure Based Biosensor For Glucose And Cholesterol Detection. Research Journal of Pharmaceutical, Biological and Chemical Sciences. 2019;10(1):150.

[38.] Samira Eissazadeh MP, Mohammad Sadegh Taskhiri, Mostafavi SM. Improvement of Sensitivity of AntigenAntibody Detection of Semen Through Gold Nanoparticle. Research Journal of Pharmaceutical, Biological and Chemical Sciences. 2019;10(1):144.

[39.] Samira Eissazadeh MP, Mostafavi SM. Measurement of Some Amino Acid Using Biosensors Based on Silicon-Based Carbon Nanotubes. Journal of Computational and Theoretical Nanoscience. 2019;16:1.

[40.] Aida Badamchi Shabestari SMM, Hanieh Malekzadeh. Force Degradation Comparative Study on Biosimilar Adalimumab and Humira. Revista Latinoamericana de Hipertensión. 2019;13(06):496-509.

\section{How to Cite This Article}

Najib Rahman, Ismaeil Rabbani, Reyhaneh Rafiei, Maryam Asadi, The Theory and Implementation of Bayesian Networks Structural Learning using Tabu Search Algorithm, Brilliant Engineering, 1(2021), 6-9. https://doi.org/10.36937/ben.2021.001.002. 